\title{
A Thresholding Approach for Pollen Detection in Images Based on Simulated Annealing Algorithm
}

\author{
Hanane Menad, EEDIS Laboratory, Djillali Liabes University, Sidi Bel Abbes, Algeria \\ Farah Ben-Naoum, EEDIS Laboratory, University of Djillali Liabes, Sidi Bel Abbes, Algeria \\ Abdelmalek Amine, GeCoDe Laboratory, Department of Computer Science, Tahar Moulay University \\ of Saida, Saida, Algeria
}

\begin{abstract}
Melissopalynology is a field that studies pollen grain origins to identify their species. It consists of studying either the chemical composition of each grain, or their shapes using microscopic images. This paper presents a system of pollen identification based on the microscopic images, it is divided into two parts, first part is the pollen detection using a thresholding method with simulated annealing algorithm. The second step is the pollen classification, in which we used deep convolutional neural network to extract features from the detected pollen grains and represent them in numerical vectors, therefore, we can use these vectors to classify them based on fully connected neural network, SVM or similarity calculation. The obtained results showed a high efficiency of the neural network in which it could recognize $98.07 \%$ of the pollen species compared not just to SVM and similarity methods, but also to works from literature.
\end{abstract}

\section{KEYWORDS}

Convolutional Neural Networks, Deep Learning, Melissopalynology, Metaheuristics, Pollen Identification, Simulated Annealing

\section{INTRODUCTION}

Image analysis systems now have an important and all-ever original place, first because they have proved their interest in areas such as image restoration or three-dimensional vision, but also because they are being justified in other previously unexplored areas 
such as diagnostic assistance. Moreover, the image represents one of the richest sources of information. Because of the variety of possibilities that this information offers, in a myriad of areas, there has been a great deal of enthusiasm for research in the field of computer vision, especially since the advent of digital images. In this regard, Numerous researches has been carried out in this field, and remains one of the most studied areas. There are basically two levels of automatic image processing. The first is a low-level treatment dedicated to acquisition, compression, segmentation, improvement or restoration. The second level is a high-level processing dedicated to symbolic image analysis operations, such as description, recognition or interpretation, in order to extract information (Benaichouche, 2014).Melissopalynology or pollen analysis of honey is one of the areas that benefited greatly from image processing and analysis techniques, where melissopalynology is the science that studies the pollen contained in honey, using a microscopic examination (Yang, 2014).In several palynology research, scientists interested in studying the distribution of pollen species, by studying the spectrogram of pollen developed for a given region from the results of several samples, to determine the botanical and geographical origin of honey, and to control the quality of honey and in particular to detect fraud and mixtures (Louveaux, 1970). Nevertheless, the identification process may be complicated because of the floral morphological similarity of many plant species, this process is more often realize manually by human expert through a visual observation on a microscope. In addition, the manual classification can be expensive to acquire for a human being because of particular physical constraints being concentration and the time required for a large volume of image data. Therefore, this process can take even months (Flenley, 1968). And because of the hardness of to the manual counting pollen and the increased use of applications of palynology, the requirement for a pollen recognition automation has become an evidence. Then in this regard, palynologists can simply take a screenshot of images observed in the microscope based on visualization system and machine learning techniques, and they can reduce time from months to hours (Scharring, 2006). Figure 1 shows the manual pollen recognition process done by biologists, in which the biologist must detect in the image the pollen which equals to make the segmentation of the image, and is based on the information observed on this pollen namely: the

Figure 1. Manual pollen recognition process done by biologists

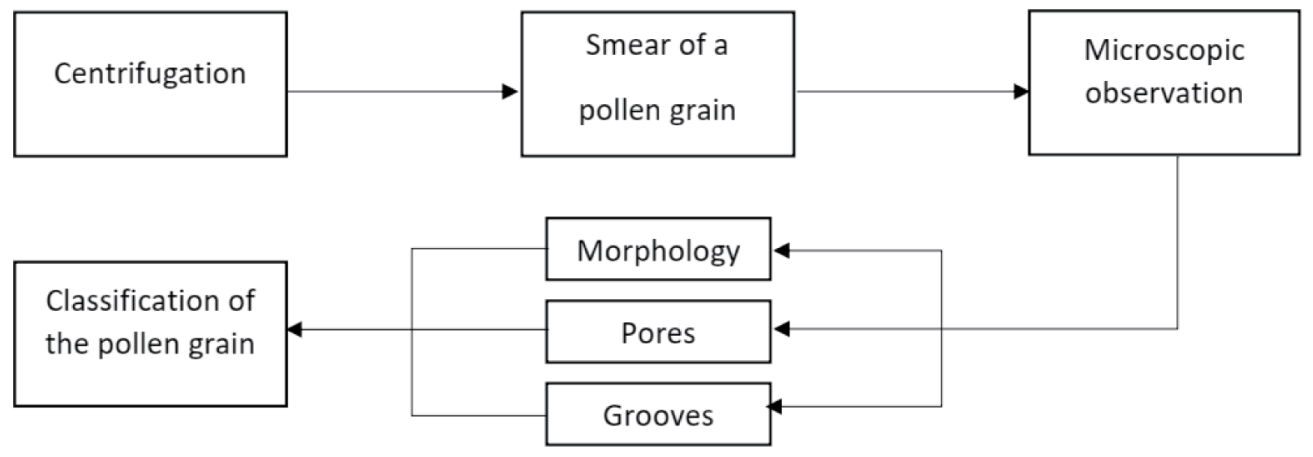


morphology or the form, the pores and grooves whose number and the provision differs from one species to another. The difficulty is that many botanical species has a similar floral morphology which makes the processes of the pollen recognition very difficult for the human.

In algorithmic, image segmentation is an image processing operation that aims to assign to each pixel of the image a label of belonging to a given region. This classification of the pixels can be carried out in a supervised or unsupervised manner. In the first case, the segmentation is performed by determining, for each site, the class whose properties are closest to those observed in it. In this case the number of regions and their characteristics are provided by the user. In the second case, the information necessary for the pixel classification process must be provided completely automatically. Hence, the complexity of this problem. Image segmentation is a large subject of study. for which various methods of segmentation are proposed in the literature. However, up to now, no image segmentation algorithm can provide perfect results on a various image (Nakib, 2007).

Recently optimization metaheuristics and image segmentation are among the most active areas of research. Indeed, the problem of image segmentation is formalized as a combinatorial optimization problem. Hence, the use of metaheuristics.

Metaheuristics method are high level optimization procedures, dedicated to solving so-called difficult optimization problems. Over the past 20 years, metaheuristics have received increasing use and have achieved success in various fields, and this is due to their capability to solve large and complex problems, these are usually problems with noisy, incomplete data, or limited computing capacity. Metaheuristics are usually inspired by biology (evolutionary algorithms), ethology (particulate swarms, ant colonies), or physics (simulated annealing) (Talbi, 2009). In fact, over the last few years, many studies have been presented with the aim to introduce metaheuristics in the field of image processing, this allowed to study the segmentation under a different angle. The results in this domain show a great improvement by proving the capacity of the proposed ideas (Siarry, 2013).

Deep learning is a set of machine learning techniques that has recognized recently great progress in the field of image recognition. It allows a computer to better recognize objects highly deformed, which made these applications more widely used in the automatic pattern recognition (LeCun, 2015).

Thresholding is one of the image segmentation methods that have been recognized great success in extracting objects from the background of the image. These methods supposed that gray levels are a measure used to distinguish among different parts of the image (Bolon, 1995).

In this view, our paper is structured as follows. In section 2, we cited some works that has relation with our study fields. Section 3 gives details about the proposed approach by giving a global architecture and describing different parts of it. Section 4 presents the obtained results, first we defined the used dataset in experiments, and then we illustrated the given results in details, after that, we compared these results with others from literature. Finally, section 5 gives major conclusions and future works. 


\section{VIEW OF LITERATURE}

Recently, pollen automatic recognition is one of the interesting and challenging tasks. Fernandez et al. in (Fernandez, 2003) compared four methods (minimum distance classifier (MDC, used in the previous work), K Nearest Neighbors (KNN), Multilayer Perceptron Neural Network (MLP), and Support Vector Machine (SVM)) for classification of 100 pollen loads collected in three places based on their texture features extracted using Local Linear Transformations, their proposed system reaches $76 \%$ of well classified species. In ( $\mathrm{Li}, 2004)$, authors conducted three different experiments, the first one they used pollen images for 4 species. In the second experiment they used 13 species with integration of their geometric information. The third experiment consisted of using texture features of the 13 species. In these experiments, authors claimed that they got very good results (up to $100 \%$ of well classified pollen species).

With the birth of new approaches called deep learning, pollen recognition was one of the first field that knew a high number of researches based on these techniques, Daood et al. (Daood, 2016) used a transfer learning-based technic to extract knowledge from pre-trained neural networks, this approach was used to recognize 30 pollen species from large datasets of images. The obtained results converged to $94 \%$. Sevillano et al. (Sevillano, 2018) presented three approaches based on convolutional neural network applied on Pollen23E dataset. The first method was the use of transfer learning to recognize pollen species. The second approach consisted of using neural network to extract features from images and then classify these features, while the second approach was a hybrid approach of the two first ones. The classification rate in this approach achieved $70 \%$.

In field of metaheuristics, simulated annealing is one the successful approaches that is used to solve many problems in various areas. In image processing, simulated annealing knew a high number of publications, for example in (Wang, 2018), simulated annealing was used for image encryption, in which it was combined with chaos algorithm, the proposed algorithm considers the generation of random sequence as a problem seeking for optimal solution and then uses the simulated annealing algorithm to further obtain the optimal pseudorandom sequences. Feng et al. (Feng, 2013) used simulated annealing algorithm to enable dynamic optimization of the cellular automata transition rules, this approach was applied to facilitate the configuration of the spatiotemporal process of urban land-use change.

\section{PROPOSED APPROACH}

Simulated annealing is a method inspired from the physical process of heating materials then slowly lowering their temperature to control the energy effects. The artificial algorithm consists of initializing a high temperature value, and then decrease this value until a stopping criterion achieved.

In this work, we proposed a thresholding method based on simulated annealing algorithm for image segmentation in order to detect pollen grains in images. The 
power of this approach is that it avoids exploring the whole image's pixels, since simulated annealing process allow moving in a manner that pixels of pollen grain have more probability to be explored. This is regard to its principle, the selection of new point in an iteration is based on probability distribution with scale proportional to the temperature. The next selected point should be a point that lower the objective using a probability, and by accepting points that raise the objective, simulated annealing explores more possible solutions rather than trapping in local minima. The temperature decreasing is done based on an annealing schedule that is selected systematically in order to reduce the extent of its search to converge to a minimum.

The selection usually is done using the following formula:

Simulated annealing value $=e^{\frac{\left(f_{\text {current }}-f_{\text {next }}\right)}{\text { temperature }}}$

where $\mathrm{f}_{\text {current }}$ is the objective function of the current position, and $\mathrm{f}_{\text {next }}$ is the objective function of the next possible position. In our case, these objective functions are the grey value of pixels since a pixel is an individual in the simulated annealing algorithm, and it is represented by its positions in line and column in the image. Figure 2 shows the architecture of the proposed simulated annealing algorithm.

As seen in Figure 2, the proposed simulated annealing algorithm takes an initial value of temperature, since we are dealing with grey value of pixels, the initial value of the temperature was the greatest grey value possible in any image which equals

Figure 2. Architecture of the proposed simulated annealing algorithm

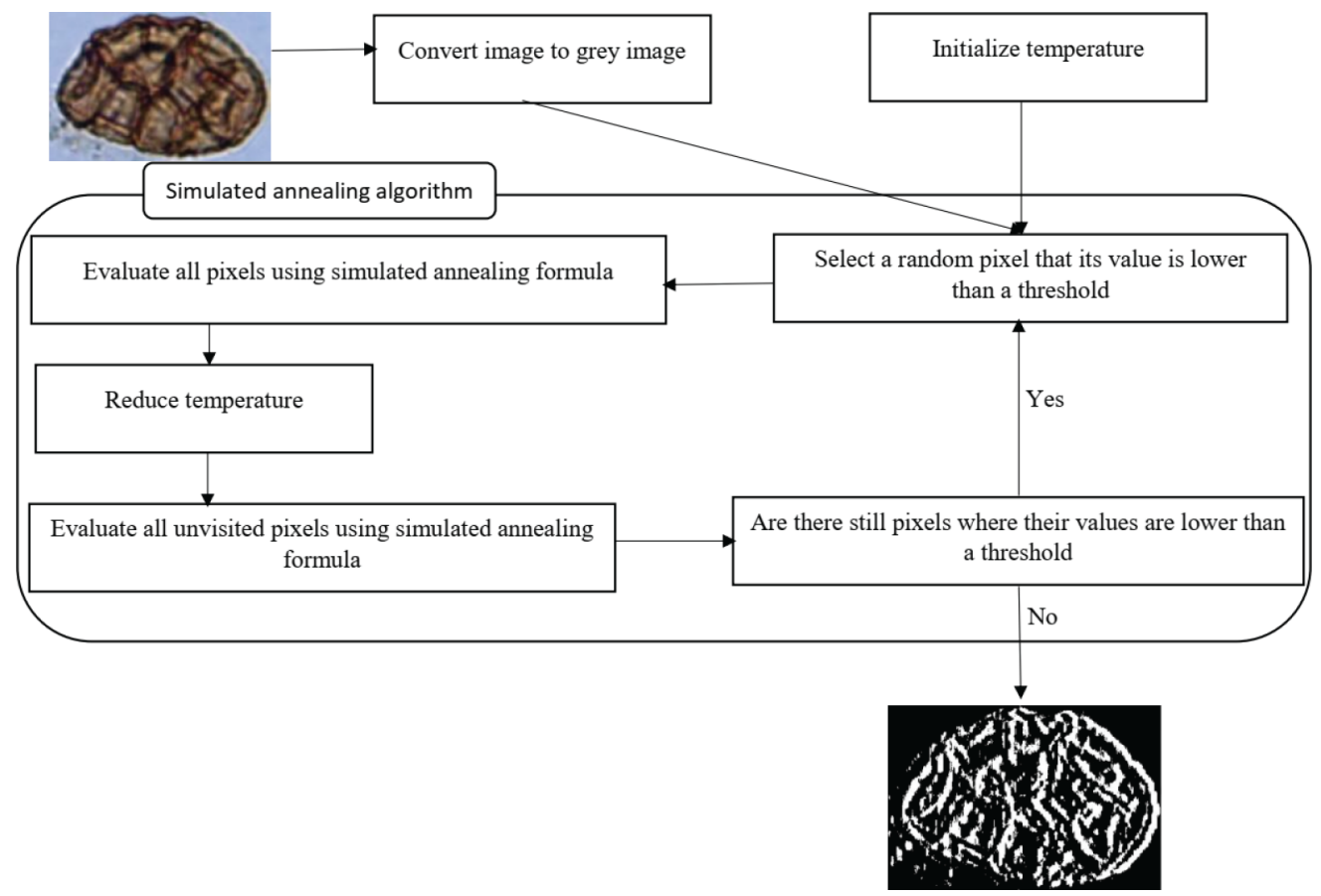


255. Then, we evaluate each pixel using simulated annealing formula, after that we choose randomly a pixel that has a value less than a fixed threshold. After choosing the pixel, we look for the next position, in which we reduce the temperature using the following formula:

Temperature $_{\text {nextPixel }}=$ Temperature $e_{\text {currentPixel }} * 0.8$

The aim of reducing the temperature is to reduce the number of possible pixels, the value 0.8 was chosen by experiments in a manner we reduced the time complexity of pollen detection. After that, we evaluate all unvisited pixels from previous iteration, then we select randomly a pixel with same condition, this process represents an iteration in the proposed simulated annealing algorithm. In other words, in each iteration of our simulated annealing, we dealt with one pixel, it only consists of selecting a pixel, evaluate it using simulated annealing formula with a threshold, and then classify it either a pollen or a background pixel. We repeat this process until a stopping criterion achieved, the stopping criterion in our case is that there are any pixels that verify the condition (simulated annealing formula $<$ threshold), finally, the selected pixels in all iteration are considered as pollen grain pixels, where the rest are background pixels. Table 1 shows examples before and after pollen detection in images.

Table 1. Examples of pollen detection results using simulated annealing algorithm

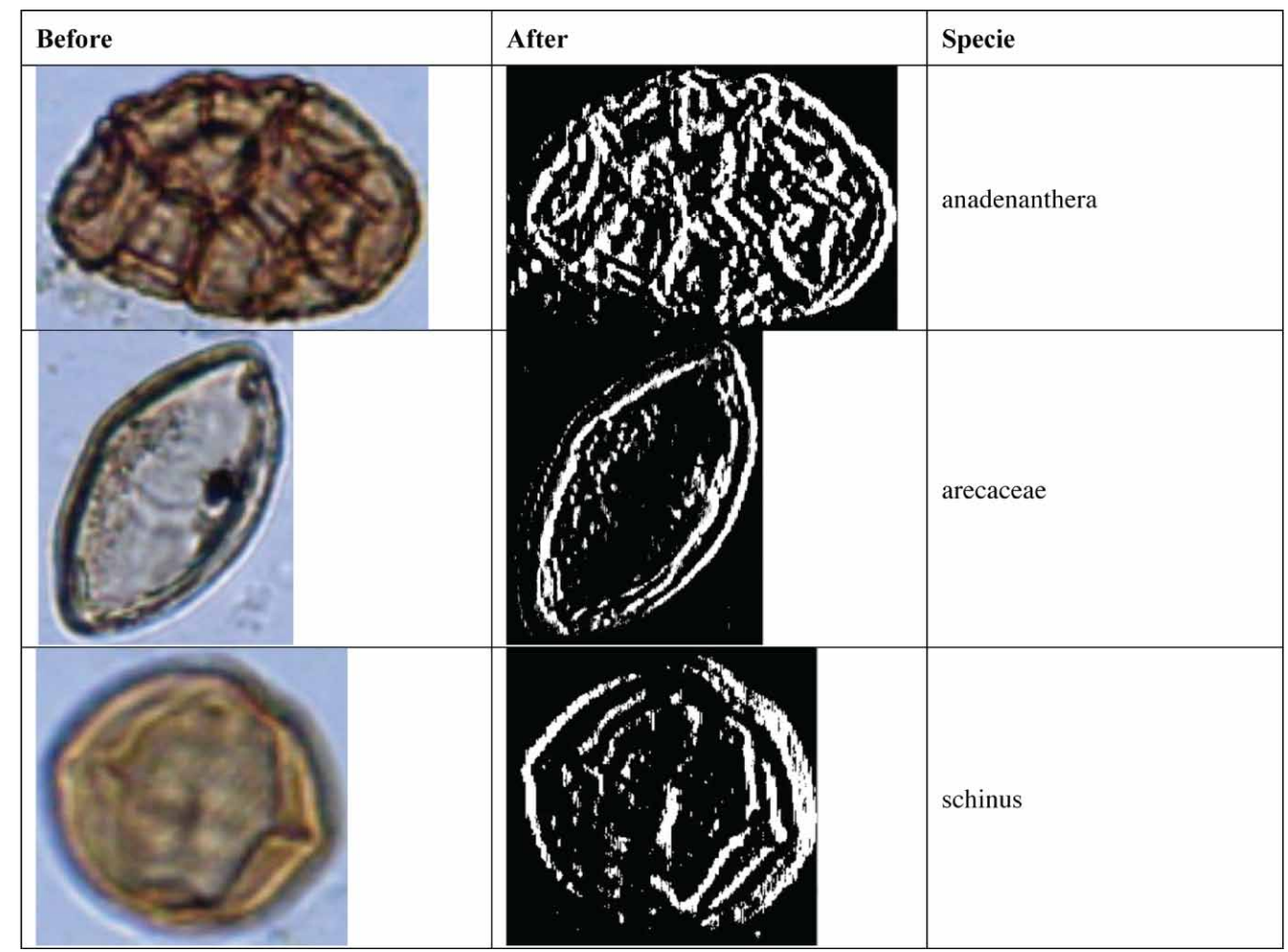


After detecting pollen grains, the resulted images are classified using deep convolutional neural network, in which we used alexNet architecture proposed by (Krizhevsky, 2012), where they used 5 layers for convolutional computation to extract features from each image using ReLu calculation and pooling for dimensionality reduction, finally they represented features extracted in 2048 vectors, and used them as input for 3 fully connected layers responsible for classification of these vectors. To retrain the model, we used 600 images from the dataset chosen randomly, and the rest were used as test set.

\section{EXPERIMENTATIONS AND RESULTS}

The following section discusses the obtained results, but before we present the used data set in experiments.

\section{Pollen23E Dataset}

Pollen23E dataset (Gonçalves, 2016) comprises a total of 35 images for each type of pollen taken at different angles. Thus, the POLEN23E dataset has 805 images. The images were captured with a digital Bresser LCD microscope at a 40x magnification. The best pictures were transferred to a laptop and segmented using the CorelDRAW1 software.

\section{Obtained Results}

In Table 2, we discuss the obtained results, in which, we based on the best training accuracy, the best validation accuracy, best cross entropy and test accuracy to evaluate the effect of number of iterations on pollen grains classification.

As seen in Table 2, number of iterations affected the obtained results in a manner that if we add more iterations, neural network builds better models. And this is clear in terms of first three measures:

Table 2. Obtained results of pollen recognition using CNN

\begin{tabular}{|l|l|l|l|l|}
\hline Number of Iteration & $\begin{array}{c}\text { Training } \\
\text { Accuracy }\end{array}$ & Cross Entropy & $\begin{array}{c}\text { Validation } \\
\text { Accuracy }\end{array}$ & Test Accuracy \\
\hline 100 & 100 & 0.71 & 89 & 90.6 \\
\hline 200 & 100 & 0.61 & 91.4 & 95.1 \\
\hline 500 & 100 & 0.26 & 91.9 & 96.6 \\
\hline 1000 & 100 & 0.14 & 93 & 96.6 \\
\hline 1500 & 100 & 0.09 & 94.42 & 97.14 \\
\hline 2000 & 100 & 0.07 & 96.5 & 97.91 \\
\hline 2500 & 100 & 0.05 & 96.99 & 98.07 \\
\hline 4000 & 100 & 0.03 & 97 & 98.07 \\
\hline
\end{tabular}


- In terms of Training accuracy: This measure is the accuracy of applying the model on the training data, it is used to evaluate the model during backpropagation steps in order to improve the model. In Table 2 and Figure 3, we cited the best training accuracy obtained, as seen, the model is perfect since it correctly classified $100 \%$ of training data. It began with a very lower accuracy then improved to reach $100 \%$, and this is because we used already trained model, then it retrained itself by adapting its weights in the fully connected layers, in other words, if we use more iterations, the model can adapt its weights better which made the pollen recognition more efficient;

- In terms of Cross Entropy: When we use cross entropy loss while training neural networks, we actually calculate the score function every time when compute gradients for the weights in the network. So, the objective is minimizing this measure, as seen in Table 2 and Figure 4, when we added more iteration, the neural

Figure 3. Training accuracy progression based on number of iterations

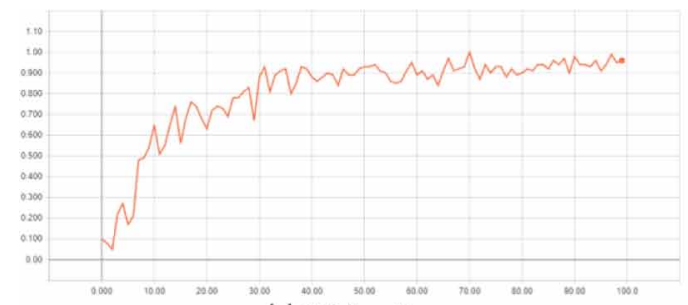

(a) 100 iteration

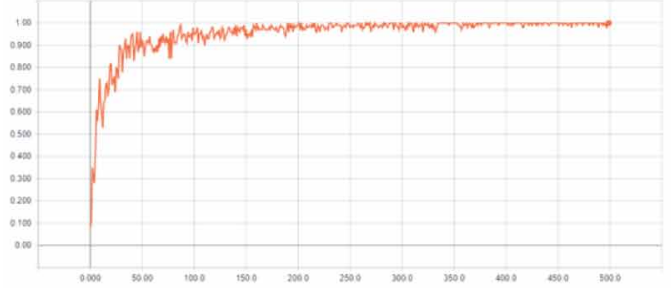

(c) 500 iteration

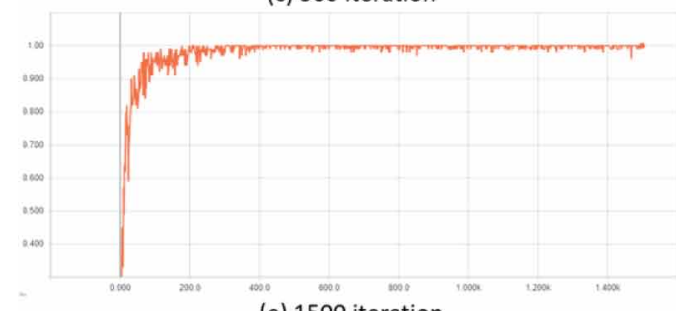

(e) 1500 iteration

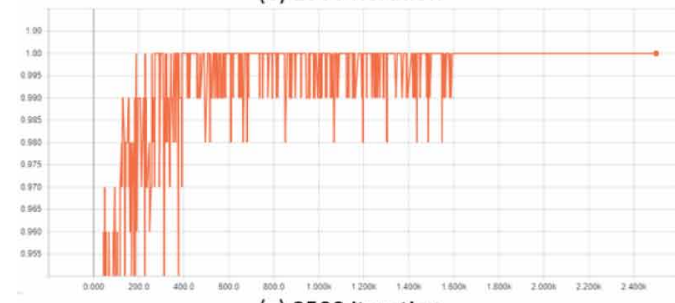

(g) 2500 iteration

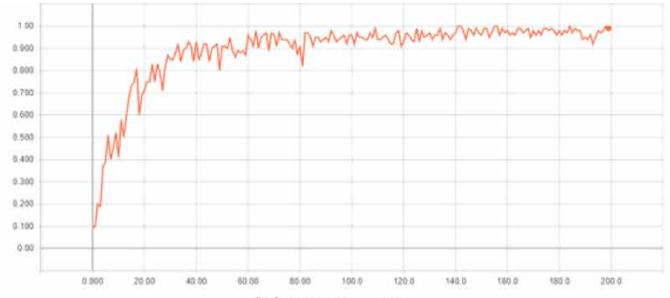

(b) 200 iteration

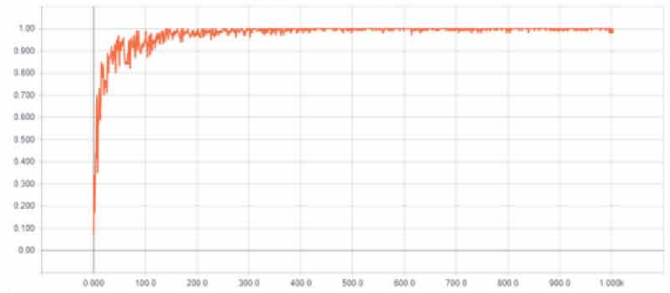

(d) 1000 iteration

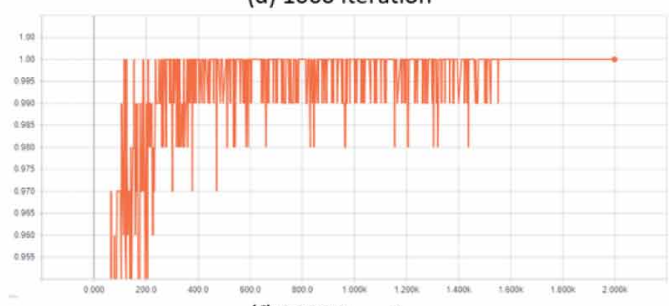

(f) 2000 iteration

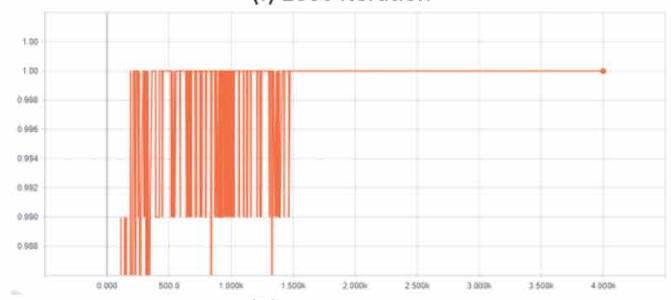

(h) 4000 iteration 
Figure 4. Cross entropy progression based on number of iterations

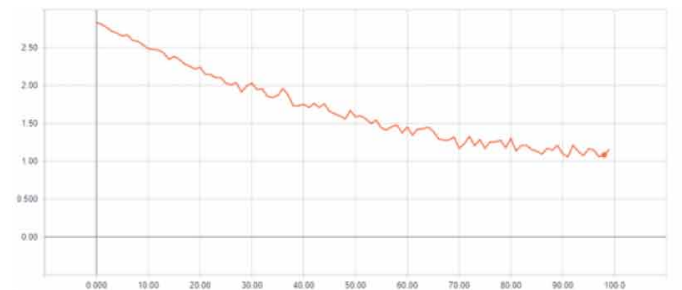

(a) 100 iteration

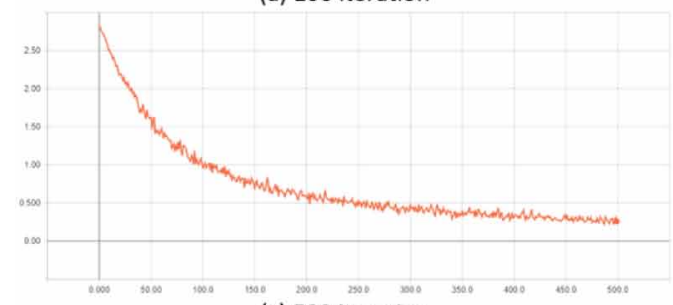

(c) 500 iteration

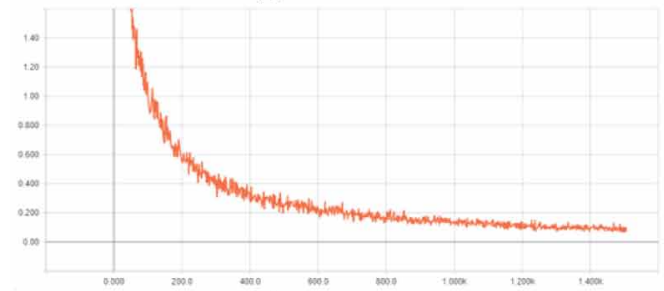

(e) 1500 iteration

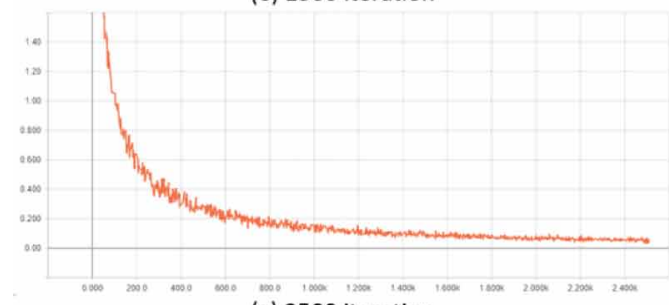

(g) 2500 iteration

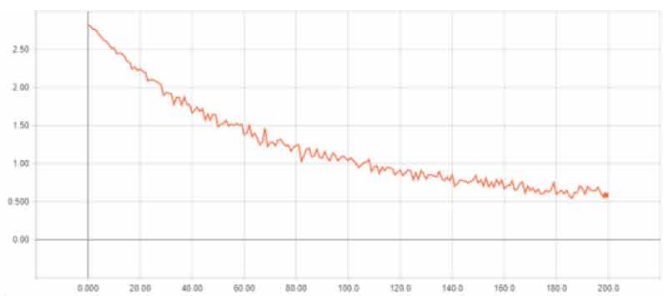

(b) 200 iteration

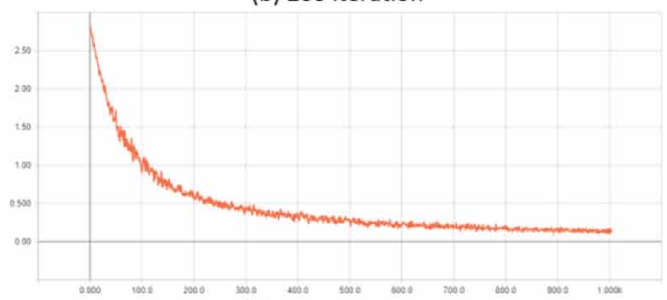

(d) 1000 iteration

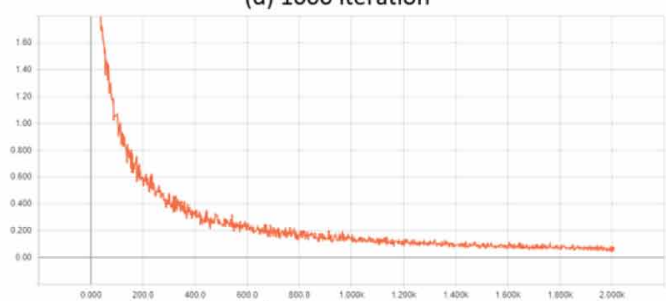

(f) 2000 iteration

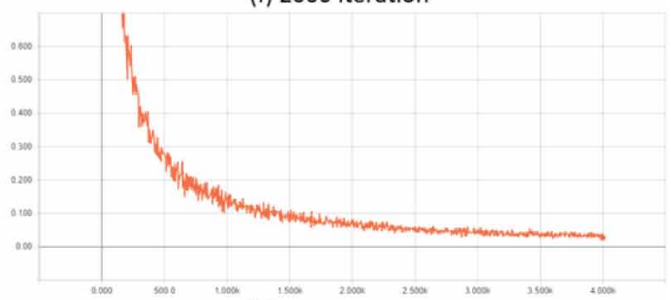

(h) 4000 iteration

network minimized the cross entropy which means we got better models. It began with a very high entropy then improved the model to decrease to 0.2 , and this is because we used already trained model, then it retrained itself by adapting its weights in the fully connected layers, in other words, if we use more iterations, the model can adapt its weights better which made the pollen recognition more efficient and minimized the information loss;

- In terms of Validation accuracy: This measure is the accuracy of applying the model on the validation data, as training accuracy, it is also used to evaluate the model during backpropagation steps in order to improve the model. In Table 2 and Figure 5, we cited the best validation accuracy obtained, as seen, the model is perfect since it correctly classified $90.6 \%$ to $100 \%$ of training data. It began with a very lower accuracy then improved to reach $100 \%$, and this is because we used already trained model, then it retrained itself by adapting its weights in the fully 
Figure 5. Validation accuracy progression based on number of iterations

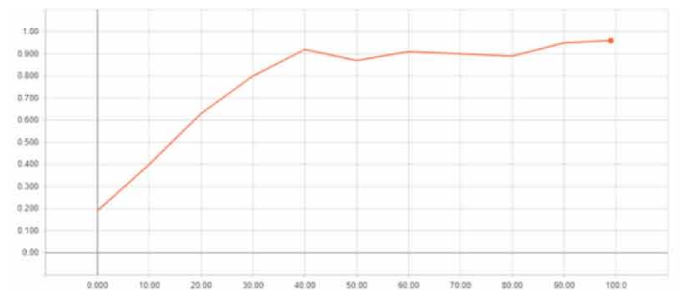

(a) 100 iteration

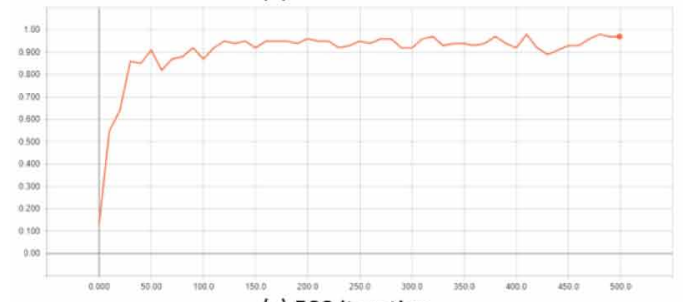

(c) 500 iteration

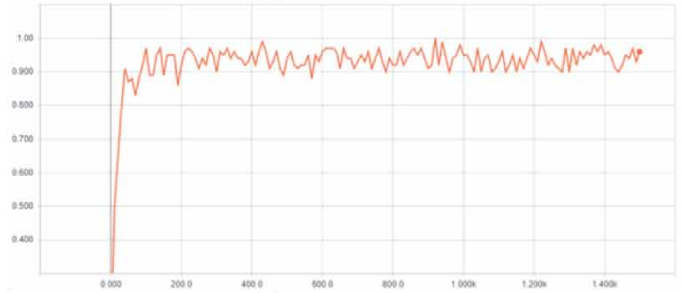

(e) 1500 iteration

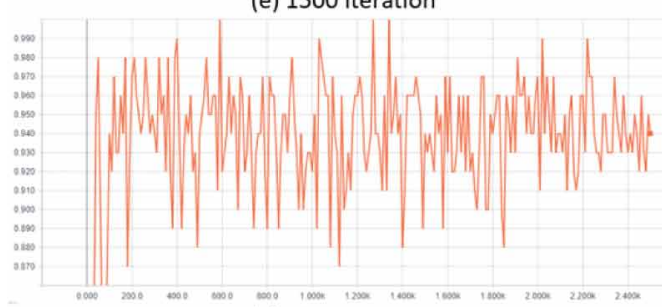

(g) 2500 iteration

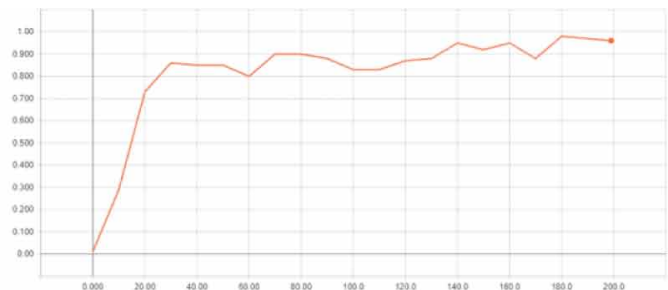

(b) 200 iteration

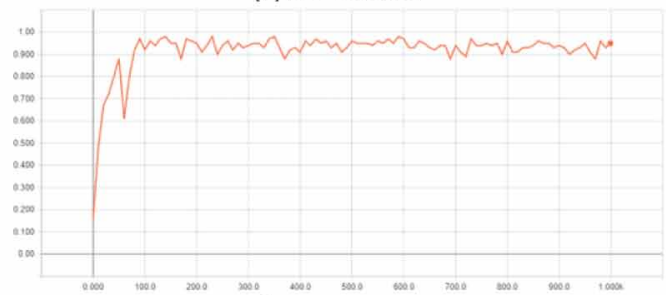

(d) 1000 iteration

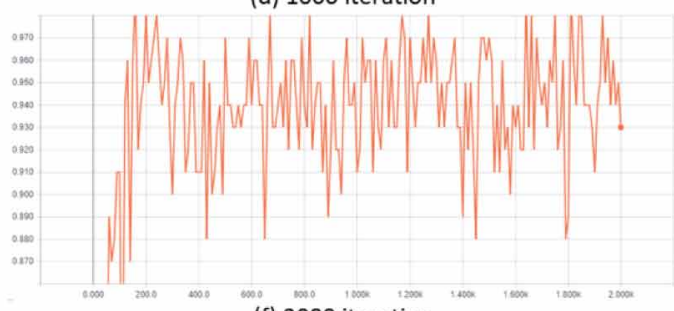

(f) 2000 iteration

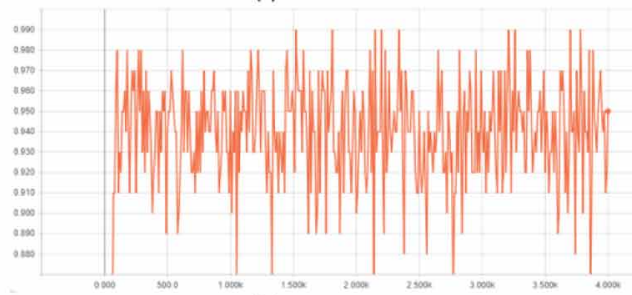

(h) 4000 iteration

connected layers, in other words, if we use more iterations, the model can adapt its weights better which made the pollen recognition more efficient;

- In terms of Test accuracy: This measure is the accuracy of applying the final model on the test data, it is used to evaluate the prediction of new images. Figure 6 shows the comparison of test accuracy according to number of iterations.

In our case Figure 6, the model recognized $90.6 \%$ of test images when we built a model in 100 iterations, while it was improved when we augmented the number of iterations to 500 iterations to recognize $96.6 \%$ up to $98.07 \%$ of pollen grains, then it became fixed despite the model has been improved based on previous measures.

To better evaluate the detection of pollen grains, we tested classification of vectors obtained using convolutional neural network from the obtained images from simulated 
Figure 6. Test accuracy according to iteration numbers

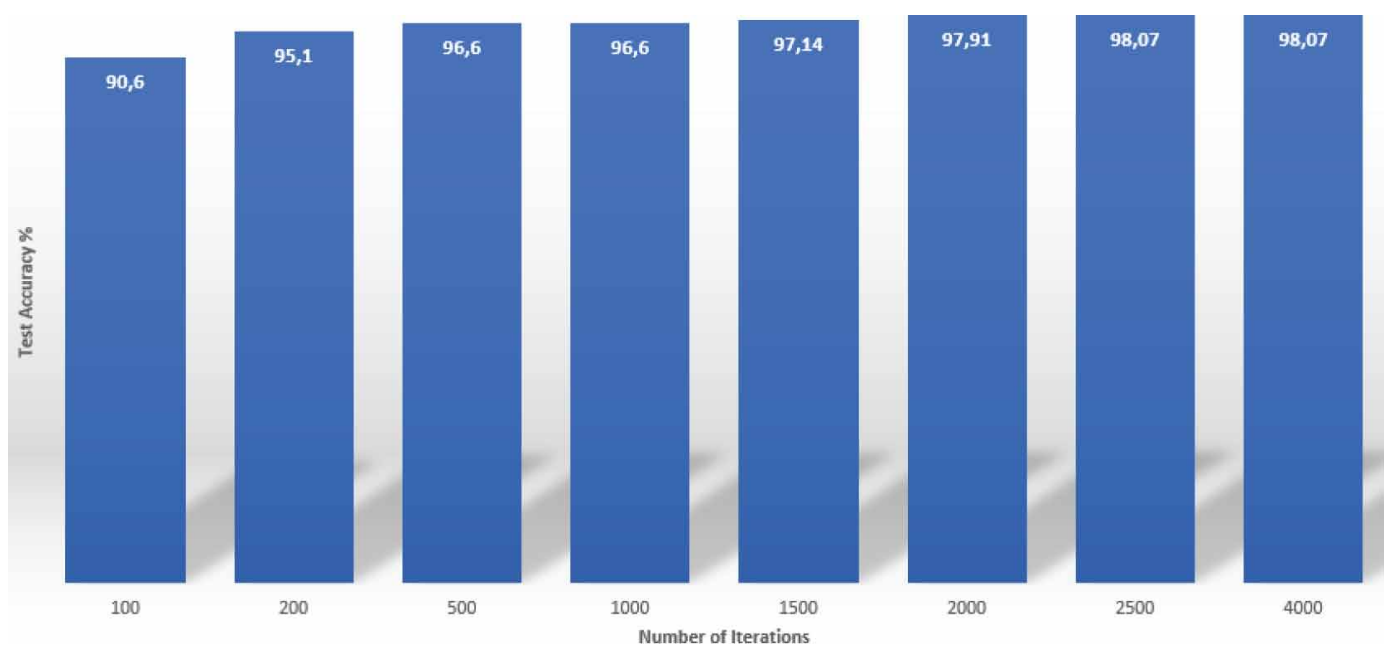

annealing, in which we used a similarity calculation between training set and test set then we used SVM algorithm for the task of classification.

For similarity calculation, we used distances are Manhattan and Euclidean:

$\operatorname{Manhattan}(\mathrm{X}, \mathrm{Y})=\sum\left|x_{i}-y_{i}\right|$

Euclidean $(\mathrm{X}, \mathrm{Y})=\sqrt{\sum\left(x_{i}-y_{i}\right)^{2}}$

where $\mathrm{x}$ is a training image, $\mathrm{y}$ is a test image, $\mathrm{x}_{\mathrm{i}}$ is the $\mathrm{i}^{\text {th }}$ pixel of the training image and $y_{i}$ is $i^{\text {th }}$ pixel of the test image.

To classify a test image, we calculated its similarity with each of training images, then we classified it by the most similar image. And for SVM, we implemented sequential minimal optimization (SMO) algorithm for training a support vector classifier using weka API. The evaluation was done based on Accuracy, Precision, Recall, and Fmeasure.

\section{Obtained Results Using Similarity and SMO for Classification}

In the experiments, we represented images using two ways, first, when simulated annealing detected a pollen pixel, it kept its grey level value, while in second experiment, we represented images using binary mask (black for background and white for pollen). Figure 7 shows the difference between the two representations of the same image in the data set.

In Table 3, we detail the obtained results of images classification of each representation using the similarity, based on measures mentioned above. Table 3 shows the comparison of different distances used to classify different representations. 
Figure 7. Representation of images from Pollen23E dataset: (a) Represents the original image; (b) Represents pollen detected with the grey level; (c) Represents pollen detected with binary mask

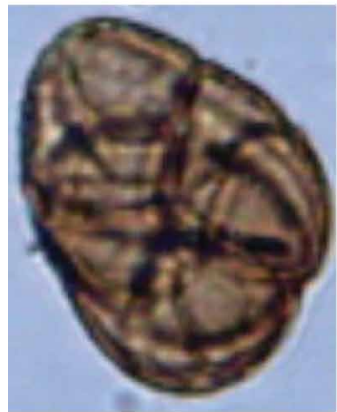

a) original image

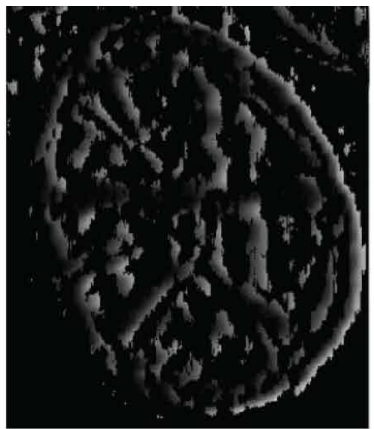

b) representation based on grey level

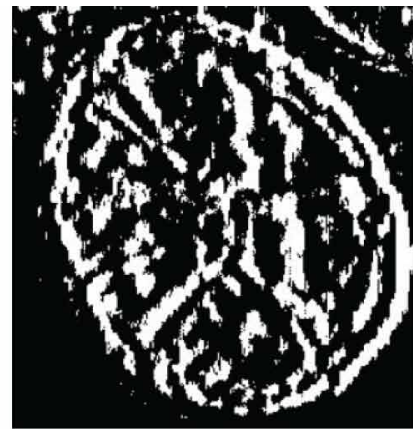

c) representation based on binary mask

Table 3. Obtained results based on distance calculation and SMO

\begin{tabular}{|l|l|l|l|l|l|}
\hline \multicolumn{1}{|c|}{ Representation } & Algorithm & Accuracy & Precision & Recall & Fmeasure \\
\hline \multirow{3}{*}{ Grey Level } & Manhattan & 87.3 & 0.872 & 0.872 & 0.872 \\
\cline { 2 - 6 } & Euclidean & 79.5 & 0.786 & 0.798 & 0.791 \\
\cline { 2 - 6 } & SMO & 93.4 & 0.933 & 0.934 & 0.934 \\
\hline \multirow{3}{*}{ Binary Masks } & Manhattan & 80.9 & 0.754 & 0.756 & 0.755 \\
\cline { 2 - 7 } & Euclidean & 80.9 & 0.754 & 0.756 & 0.755 \\
\cline { 2 - 7 } & SMO & 88.2 & 0.883 & 0.881 & 0.882 \\
\hline
\end{tabular}

As seen on Table 3, Manhattan distance gave better results than Euclidean distance, the results proved the efficiency of image segmentation in differentiating between images represented using grey level, while the two distances gave the same results when we represented the images using binary masks, that was because binary mask representation means that a pixel has only two values, either 0 or 1 . While using grey level representation, we gave an interval for each pixel from 0 to 255 , which helped Manhattan distance to find differences among images more precisely:

- In terms of accuracy: Manhattan distance recognized $87.3 \%$ of the images correctly when we represented them using grey level representation, but Euclidean distance could recognize only $79.5 \%$ of all images, which was remarkable;

- In terms of Precision: Precision is a measure that was used to know how well the system was responding to a request from new classifications of pollen grains. In our case, we have calculated the weighted precision average, which shows how well our system could predict the specie of a pollen grain that reached 0.933 of precision when we classified images represented using grey level and SMO algorithm, but when we represented images using binary masks, the results decreased to 0.883 ; 
- In terms of Recall: Recall is a measure that was used to know how well the system was responding to a request from the original classifications of pollen grains. In our case, when we represented images using binary masks, the two distances could classify correctly $75.6 \%$ from the original classification of all images, while using grey level-based representation improved the obtained recall to reach 0.798 using Euclidean distance for classification, and 0.872 using Manhattan distance;

- In terms of Fmeasure: Fmeasure is a metric used to measure how well the system can recognize elements according to a request from both new classifications and original classifications, in other words, the fmeasure combines precision and recall based on the harmonic mean of the two. Because Fmeasure depends on precision and recall, its values were related to them. The obtained fmeasure was vary from 0.791 obtained for classification of grey level-based pollen grains representation using Euclidean distance, up to 0.934 using SMO to classify the same images with grey-level representation.

These obtained results showed the efficiency the Manhattan distance compared to Euclidean distance, and this due to the dimensionality of the vectors, since we did not apply any dimensionality reduction algorithm, also all images were resized to $368 \times 368$, our vectors dimensionality contained 135424 values. The reason for this is quite simple to explain. We know that the two distances belong to Minkowski distances given as follows:

$\operatorname{Minkowski}(\mathrm{X}, \mathrm{Y})=\left(\sum\left|x_{i}-y_{i}\right|^{n}\right) \frac{1}{n}$

where $\mathrm{X}$ and $\mathrm{Y}$ are high dimensionality vectors.

If we consider that $\mathrm{n}=\infty$, then the distance is the highest difference between any two dimensions of the vectors. So, in this case, we can see that when we add more attributes to the dataset, most of them will be ignored if $n$ is greater. So, for vectors with high dimensionality, by reducing the value of the exponent $n$, we give to features a bigger role in the distance calculation. A high difference in some given dimension will be less relevant if the exponent is lower.

\section{COMPARATIVE STUDY}

To better validate our proposed approach, we compared it with results obtained by (Gonçalves, 2016), where authors used two feature extraction techniques for representation of Pollen23E species: Color, Shape and Texture (CST), Bag of Visual Words (BOW), and their combination. For evaluation, they used four supervised techniques Sequential Minimal Optimization algorithm for training a Support Vector Machine (SMO), Support Vector Machine (C-SVC), K Nearest Neighbors (KNN), and Decision Tree(C4.5). They found that combination of the two feature extraction techniques gave the best results using support vector machine-based approaches (SMO 
and C-SVC). Figure 8 shows the comparison between previously obtained results and those obtained by our approach in terms of fmeaure.

Also, we compared the obtained results with (Arias, 2017), in which authors extracted 3 features (Local binary patterns (LBP), autocolor correlograms (ACC)), and Weber Local Descriptor (WLD) from Pollen23E, authors used 4 approaches for classification of each extracted characteristic (SVM, Random Forest, logistic regression, and ensemble classifiers), and in the second table we see the result obtained after the combination of each Descriptor with 4 classifiers used the comparison was done based on the accuracy. Figure 9 shows the comparison of the best obtained accuracy by our proposed approach and the best accuracy obtained in (Arias, 2017).

As seen in Figure 8 and Figure 9, our proposed approach achieved better results than those obtained in literature, Gonçalves et al. in (Gonçalves, 2016) used feature

Figure 8. Comparison of the obtained fmeasure with fmeasure obtained in (Gonçalves, 2016)

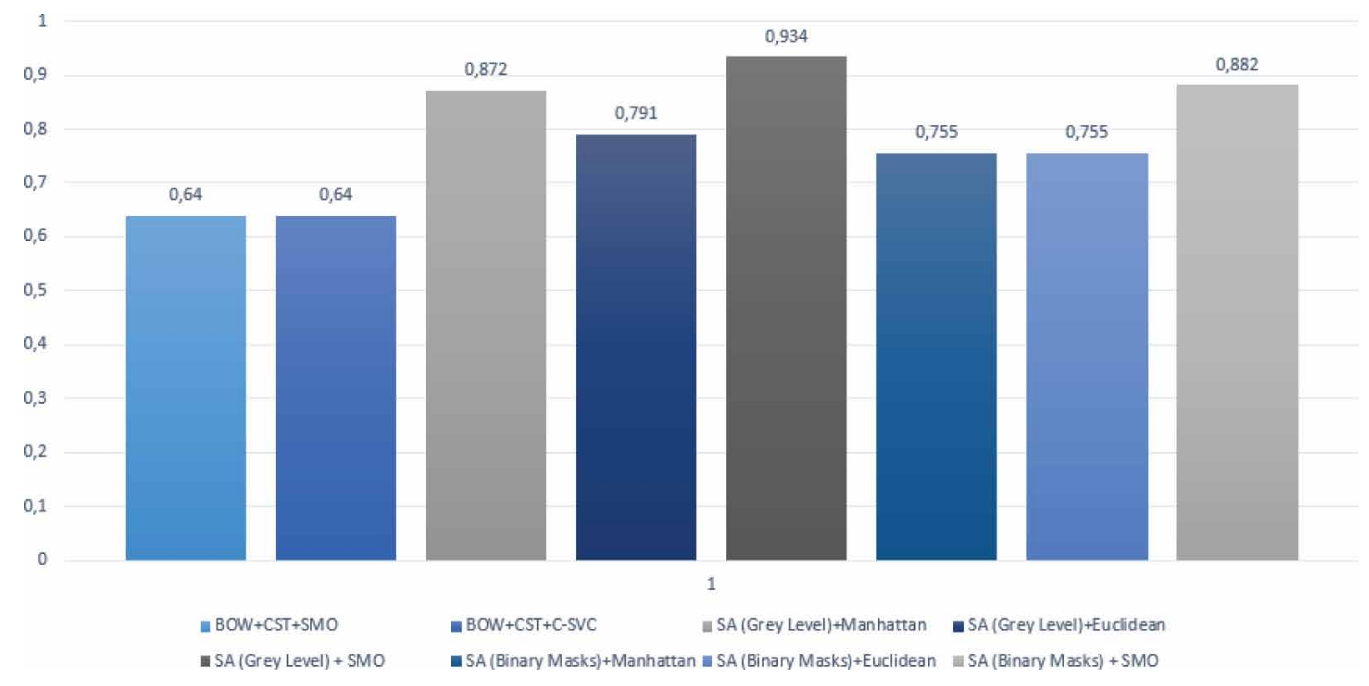

Figure 9. Comparison of the obtained accuracy with accuracy obtained in (Arias, 2017)

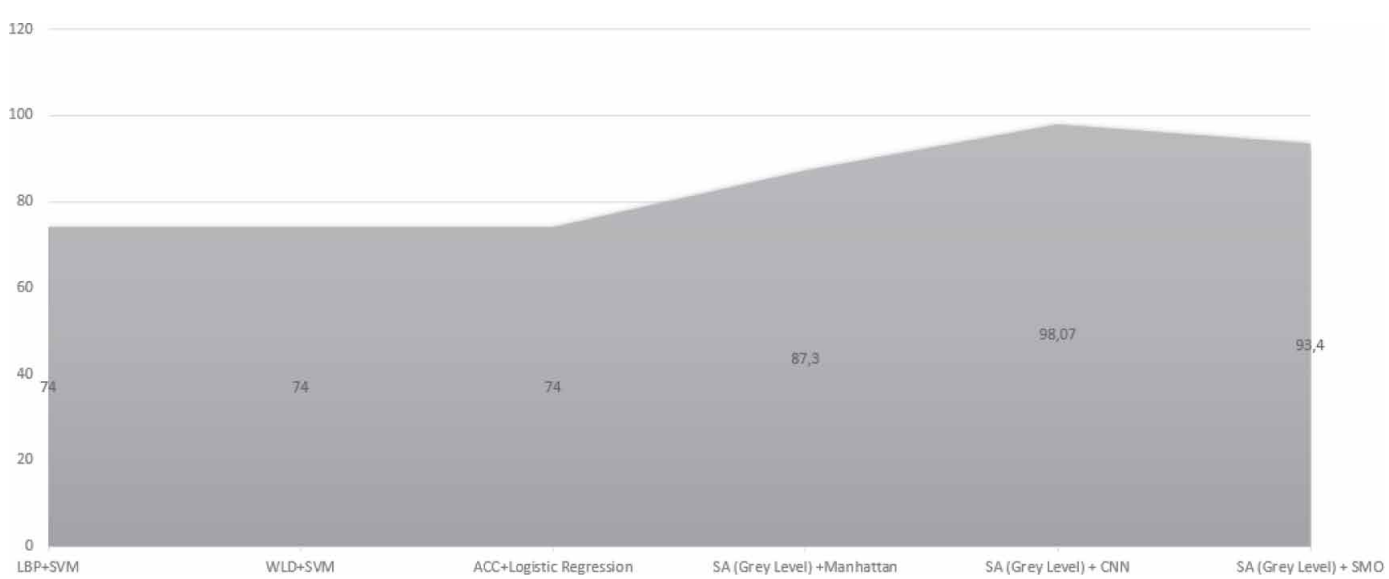


extraction techniques to get colors, shape and textures of pollen grains from images, while (Gonçalves, 2016) used approaches based on descriptor extraction by getting three descriptors. These two techniques caused information loss, especially for species that have colors slightly similar to the background, while our approach generated a threshold based on grey level of each image. The detection of pollen grain was than easy and by doing that we minimized information loss. As a result, a simple similaritybased algorithm could correctly predict about $83 \%$ of the pollen species, and when we used SMO for classification, the system recognized $87.3 \%$ of the pollen images correctly. In Figure 8 we compared our obtained results with the ones obtained by (Arias, 2017), also the Figure 9 proved the efficiency of our system, since CNN and SMO could predict more than $90 \%$ of the pollen species while techniques proposed by (Arias, 2017) could predict $74 \%$ of the species.

One of the most recent works was done by Sevillano et al. (Sevillano, 2018) where authors used the convolutional neural network for classification of pollen grain images. They used three different setups, the first one was based on feature extraction and linear discriminant classifier (FE+LD), this approach consists of extracting features from images using pre-trained AlexNet neural network, then classify these vectors using linear discriminant classifier. The second setup was called transfer learning, In this setup, the pre-trained CNN AlexNet has been adjusted to learn the particular features of the POLLEN23E images dataset by replacing the last three layers by other fully connected layers for prediction 23 classes of Pollen23E. The third setup was a hybrid approach of the tow setups mentioned where authors used retrained CNN for extraction of features from its fully connected layers then classify them using linear discriminant classifier. Since Sevillano et al. used Pollen23E dataset in their experiments, we chose to compare our obtained results from the CNN approach with their obtained results since they have used original images without detecting the pollen in them, especially the setup B, which is the same CNN used in our experiments. Table 4 shows the comparison based on accuracy and training time given knowing that all approaches were reimplemented in the same conditions (machine).

As seen in Table 4, accuracy obtained by our proposed approach $(90.6 \%$ up to 98.07\%) converged to results given by Sevillano et al. (95.5\% up to 96.3\%) knowing that for Sevillano approach, we used 4000 iteration in CNN. Despite Sevillano gave better results, we have improved the training time in our approach, that is because Sevillano used linear discriminant classifier which took more time for training a model that can predict pollen grain species. The table proved that using our approach, we need 2500 iteration in almost 8 minutes to train CNN for prediction of $98.07 \%$ of correctly, while Sevillano needs at least 11 minutes to build the model that can predict $95.5 \%$ correctly.

\section{CONCLUSION}

Melissopalynology is a field that consists of studying the pollen geographical location and its origins from genus of plants visited by bees. In this paper, we presented a 
Table 4. Comparison of the obtained results by our proposed approach and results given by Sevillano et al. (Sevillano, 2018)

\begin{tabular}{|l|l|l|}
\hline \multicolumn{1}{|c|}{ Algorithm (CNN) } & \multicolumn{1}{c|}{ Accuracy (\%) } & Training Time (min) \\
\hline Our proposed approach (100 iteration) & 90.6 & 1.10 \\
\hline Our proposed approach (200 iteration) & 95.1 & $\mathbf{2 . 0 2}$ \\
\hline Our proposed approach (500 iteration) & 96.6 & 2.57 \\
\hline $\begin{array}{l}\text { Our proposed approach (1000 } \\
\text { iteration) }\end{array}$ & 96.6 & 4.52 \\
\hline $\begin{array}{l}\text { Our proposed approach (1500 } \\
\text { iteration) }\end{array}$ & 97.14 & 6.03 \\
\hline $\begin{array}{l}\text { Our proposed approach (2000 } \\
\text { iteration) }\end{array}$ & 97.91 & 7.3 \\
\hline $\begin{array}{l}\text { Our proposed approach (2500 } \\
\text { iteration) }\end{array}$ & $\mathbf{9 8 . 0 7}$ & 7.59 \\
\hline $\begin{array}{l}\text { Our proposed approach (4000 } \\
\text { iteration) }\end{array}$ & $\mathbf{9 8 . 0 7}$ & 11 \\
\hline Sevillano et al. (Setup A) (FE+LD) & 95.5 & 11.52 \\
\hline Sevillano et al. (Setup B) (TL) & 95 & 17.01 \\
\hline $\begin{array}{l}\text { Sevillano et al. (Setup C) } \\
\text { (TL+FE+LD) }\end{array}$ & $\mathbf{9 6 . 3}$ & 17.18 \\
\hline
\end{tabular}

system for recognition of pollen grains based on two steps, first, we detected pollen grains in images using a thresholding technic based on simulated annealing algorithm, then, we classified the detected pollen using deep convolutional neural network that was divided into 8 layers ( 5 convolutional layers to extract features from images, and 3 fully connected layers to classify the obtained features). Features extraction was done pooling and ReLU non linearities convolutional computation.

By applying classical thresholding methods, we move among pixels randomly, so we have risks of passing by the same pixel many times, in our case we avoided that. Also, by using simulated annealing algorithm, we can say that we cluster each pixel (classify it either pollen or background pixel) in one iteration, while using k-means algorithm, we have to choose a pollen pixel and background pixel as centers, and then we cluster the pixels in many iterations until both clusters are fixed. In other words, we optimized the pollen detection complexity compared to k-means algorithm.

The obtained results proved the efficiency of deep convolutional neural networks for pollen grains classification, which is clear in terms of training accuracy, validation accuracy and cross entropy, in other hand, we saw that the proposed approach avoid the underfitting, this is proved by the validation accuracy that was lower than training accuracy. Also, we discussed the use of supervised classification techniques (SMO and similarity-based classification) for image classification to predict the specie of each pollen, but first, we represented the detected pollen grains using two methods, grey-level based representation where we kept grey value of each pixel, and a binary mask-based technique, where a pixel could have only two values ( 1 or 0 ). The proposed 
approach proved its efficiency in pollen recognition in terms of four performance measures (accuracy, precision, recall and fmeasure). Also, compared to results from the literature, Simulated annealing proved that is more suitable for pollen classification. Its disadvantage is laying on the complexity, more the image is bigger, more time our approach takes to detect the pollen grain.

For future works, we planned to develop more approaches for image segmentation is planned. In our experiments, we did not use a reduction of dimensionality methods, we just classified images by their original sizes, which took a lot of time. So, our main work is including such techniques to reduce dimensions of images in the global proposed approach. 


\section{REFERENCES}

Arias, D. G., Cirne, M. V. M., Chire, J. E., \& Pedrini, H. (2017, December). Classification of Pollen Grain Images Based on an Ensemble of Classifiers. In Proceedings of the 2017 16th IEEE International Conference on Machine Learning and Applications (ICMLA) (pp. 234-240). IEEE. doi:10.1109/ICMLA.2017.0-153

Benaichouche, A. N. (2014). Conception de métaheuristiques d'optimisation pour la segmentation d'images: application aux images IRM du cerveau et aux images de tomographie par émission de positons [Doctoral dissertation]. Université Paris-Est.

Bolon, P., Chassery, J. M., Cocquerez, J. P., Demigny, D., Graffigne, C., Montanvert, A., \& Maître, H. (1995). Analyse d'images: filtrage et segmentation. Masson.

Daood, A., Ribeiro, E., \& Bush, M. (2016, December). Pollen grain recognition using deep learning. In Proceedings of the International Symposium on Visual Computing (pp. 321-330). Springer.

Feng, Y., \& Liu, Y. (2013). A heuristic cellular automata approach for modelling urban land-use change based on simulated annealing. International Journal of Geographical Information Science, 27(3), 449-466. doi:10.1080/13658816.2012.695377

Fernandez-Delgado, M., Carrion, P., Cernadas, E., Galvez, J. F., \& Sa-Otero, P. (2003). Improved classification of pollen texture images using SVM and MLP. In Proceedings of the international conference on visualization, imaging, and image processing (Vol. 2).

Flenley, J. R. (1968). In M. B. Clowes \& J. P. Penny (Eds.), The problem of pollen recognition. In Problems in Picture Interpretation (pp. 141-145). Canberra: CSIRO.

Gonçalves, A. B., Souza, J. S., da Silva, G. G., Cereda, M. P., Pott, A., Naka, M. H., \& Pistori, H. (2016). Feature Extraction and Machine Learning for the Classification of Brazilian Savannah Pollen Grains. PLoS One, 11(6), e0157044. doi:10.1371/journal. pone.0157044 PMID:27276196

Krizhevsky, A., Sutskever, I., \& Hinton, G. E. (2012). Imagenet classification with deep convolutional neural networks. In Advances in neural information processing systems (pp. 1097-1105).

LeCun, Y., Bengio, Y., \& Hinton, G. (2015). Deeplearning. nature, 52 1(7553), 436.

Li, P., Treloar, W. J., Flenley, J. R., \& Empson, L. (2004). Towards automation of palynology 2: The use of texture measures and neural network analysis for automated identification of optical images of pollen grains. Journal of Quaternary Science, 19(8), 755-762. doi:10.1002/jqs.874

Louveaux, J., Maurizio, A., \& Vorwohl, G. (1970). Commission internationale de botanique apicole de l'uisb: les méthodes de la mélisso-palynologie. Apidologie, 1(2), 211-227. doi:10.1051/apido:19700206 
Nakib, A. (2007). Conception de mtaheuristiques d'optimisation pour la segmentation d'images. Application aux images biomdicales [Doctoral dissertation]. Universit Paris XII Val de Marne.

Scharring, S., Schultz, E., Heimann, U., Gehrig, R., Defila, C., Kühler, B., ... \&Sulz, G. (2006). Automatic Pollen Recognition-Developments and Perspectives. Nachrichtenblatt des DeutschenPflanzenschutzdienstes, 58.

Sevillano, V., \& Aznarte, J. L. (2018). Improving classification of pollen grain images of the POLEN23E dataset through three different applications of deep learning convolutional neural networks. PLoS One, 13(9), e0201807. doi:10.1371/journal. pone.0201807 PMID:30216353

Siarry, P. (Ed.). (2013). Optimisation in signal and image processing. John Wiley \& Sons.

Talbi, E. G. (2009). Metaheuristics: from design to implementation (Vol. 74). John Wiley \& Sons. doi:10.1002/9780470496916

Wang, X., Liu, C., Xu, D., \& Liu, C. (2016). Image encryption scheme using chaos and simulated annealing algorithm. Nonlinear Dynamics, 84(3), 1417-1429. doi:10.1007/ s11071-015-2579-y

Yang, Y. (2014). Qualification des miels de Corse par une approche multifactorielle: diversité pollinique \& variabilité chimique [Doctoral dissertation]. Université de CORSE-Pascal PAOLI.

Hanane Menad received a license degree in computer Science from the Computer Science department of Dr. Tahar Moulay University of Saida-Algeria, received a Master diploma in Data mining and knowledge management from same University. Now, Hanane Menad is a PhD student. Her research interests include data mining, knowledge extraction, bioinspired algorithms, medical data analysis, melissopalynology data processing.

Farah Ben-Naoum received the engineer degree in computer status, the master's degree, the PhD degree and HDR in artificial intelligence from Djillali Liabès University, Algeria, in 1997, 2002, 2010 and 2016, respectively. Now, she is a professor and researcher at the Department of Computer Science of Djillali Liabès University. Since 2003, she has been a member of the Evolutionary Engineering and Distributed Information Systems Laboratory. Her research interests include architectural plant modeling, tree compression, tree editing distance and heuristics for bioinformatic.

Abdelmalek Amine received an engineering degree in Computer Science, a Magister diploma in Computational Science and PhD from Djillali Liabes University in collaboration with Joseph Fourier University of Grenoble. His research interests include bigdata, IoT, data mining, text mining, ontology, classification, clustering, neural networks, and biomimetic optimization methods. He participates in the program committees of several international conferences and on the editorial boards of international journals. Prof. Amine is the head of GeCoDeknowledge management and complex data-laboratory at UTM University of Saida, Algeria; he also collaborates with the "knowledge base and database" team of TIMC laboratory at Joseph Fourier University of Grenoble. 